\title{
Does PET Reconstruction Method Affect Deauville Score in Lymphoma Patients?
}

\author{
Blandine Enilorac ${ }^{1}$, Charline Lasnon ${ }^{2}$, Cathy Nganoa $^{1}$, Christophe Fruchart $^{3}$, Anne-Claire Gac $^{3}$, Gandhi Damaj ${ }^{3}$, \\ and Nicolas Aide ${ }^{1,4}$ \\ ${ }^{1}$ Nuclear Medicine Department, Caen University Hospital, Caen, France; ${ }^{2}$ Nuclear Medicine Department, François Baclesse Cancer \\ Centre, Caen, France; ${ }^{3}$ Haematology Institute, Caen University Hospital, Caen, France; and ${ }^{4}$ INSERM 1199 ANTICIPE, Normandie \\ University, Caen, France
}

\begin{abstract}
When evaluating ${ }^{18} \mathrm{~F}-\mathrm{FDG}$ PET images with the Deauville score (DS), the quantification of tumor and reference organs limits the problem of optical misinterpretation. Compared with conventional reconstruction algorithms, point-spread function (PSF) modeling increases SUVs significantly in tumors but only moderately in the liver, which could affect the DS. We investigated whether the choice of the reconstruction algorithm affects the DS and whether discordance affects the capability of ${ }^{18} \mathrm{~F}-\mathrm{FDG}$ PET to stratify lymphoma patients. Methods: Overall, 126 patients with diffuse large B-cell lymphoma were included (56 female and 70 male; median age, $65 \mathrm{y}$; range, 20-88 y). PET data were reconstructed with the unfiltered PSF method. Additionally, a 6-mm filter was applied to PSF images to meet the requirements of the EANM Research Ltd. (EARL) harmonization program from the European Association of Nuclear Medicine (EANM) $\left(\right.$ PSF $_{\text {EARL }}$ ). One hundred interim PET (i-PET) and 95 end-of-treatment PET (EOT-PET) studies were analyzed. SUV $\max$ in the liver and aorta was determined using automatic volumes of interest and compared with SUV $\mathrm{Sax}_{\text {in }}$ in the residual mass with the highest ${ }^{18} \mathrm{~F}-\mathrm{FDG}$ uptake. Results: For i-PET, using PSF and

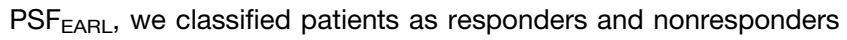
in 60 and 40 cases versus 63 and 37 cases, respectively. Five cases of major discordance (5.0\%) occurred (i.e., changes from responder to nonresponder). For Eot-PET, patients were classified using PSF and PSF $_{\text {EARL }}$ as responders and nonresponders in 69 and 26 cases versus 72 and 23 cases, respectively. Three cases of major discordance (3.2\%) occurred. Concordance (Cohen unweighted $\mathrm{k}$ ) between the PSF and the PSF EARL $_{\text {DS }}$ was $0.82(95 \%$ confidence interval, 0.73-0.91) for i-PET and 0.89 (95\% confidence interval, 0.81-0.96) for EOT-PET. The median follow-up periods were 28.4 and 27.4 mo for i-PET and EoT-PET, respectively. Kaplan-Meier analysis showed statistically significant differences in progressionfree survival and overall survival among responders and nonresponders no matter which reconstruction was used for i-PET and EOT-PET. Conclusion: Neither DS nor risk stratification of diffuse large B-cell lymphoma patients is affected by the choice of PET reconstruction. Specifically, the use of PSF is not an issue in routine clinical processes or in multicenter trials. These findings have to be confirmed in escalation and deescalation procedures based on early i-PET.
\end{abstract}

\footnotetext{
Received Sep. 27, 2017; revision accepted Nov. 28, 2017.

For correspondence or reprints contact: Nicolas Aide, Nuclear Medicine Department, Caen University Hospital, Avenue Cote de Nacre, Caen 14000, France.

E-mail: aide-n@chu-caen.fr

Published online Dec. 14, 2017.

COPYRIGHT (C 2018 by the Society of Nuclear Medicine and Molecular Imaging.
}

Key Words: PET; lymphoma; Deauville score; interim; end-oftreatment; reconstruction algorithm

J Nucl Med 2018; 59:1049-1055

DOI: 10.2967/jnumed.117.202721

I the staging, monitoring, and restaging of Hodgkin and nonHodgkin lymphoma patients, ${ }^{18} \mathrm{~F}$-FDG PET has become the standard procedure (1). During therapy assessment at mid treatment (interim PET [i-PET]) and after completion of chemotherapy (end-of-treatment PET [EoT-PET]), the Deauville score (DS) is used to discriminate between responders and nonresponders (2-4). Responders are usually defined as DS1-DS3, whereas nonresponders are defined as DS4 and DS5, except for deescalating trials in which DS2 may be required at i-PET for entry into the deescalated arm $(1,5)$. Quantitative evaluation of residual tumor masses and reference organs (liver and blood pool) is required when scoring ${ }^{18}$ F-FDG PET with the DS because this approach limits the problem of optical misinterpretation due to the influence of background activity $(1,4)$.

Over the past few years, new reconstruction algorithms have been released and shown to improve diagnostic accuracy in various solid tumors. In particular, point-spread function (PSF) reconstruction is available from the 3 major PET vendors and is progressively replacing conventional ordered-subset expectation maximization either alone or in addition to time-of-flight (TOF) capability (6). In addition to the improvement in diagnostic performance (7), PSF modeling significantly increases SUV metrics in tumor lesions compared with conventional reconstruction algorithms, but only moderately in the liver and in the vascular background $(8,9)$. Consequently, these increases could affect DS by systematically increasing the score. This issue was exemplified by the RATHL lymphoma trial, which mandated that centers with PSF reconstruction or TOF disable these features when participating in the study (10). The EANM Research Ltd. (EARL) harmonization program from the European Association of Nuclear Medicine (EANM) has been shown to efficiently overcome the issue of reconstruction inconsistencies in nonhematologic solid tumors (11). EARL-accredited centers tend to use 2 PET datasets: one optimized for diagnostic purposes and one using a filter chosen so that the reconstruction meets the EANM/EARL harmonizing standards.

The aim of the present study was to investigate whether the choice of reconstruction algorithm may affect DS in a significant 
TABLE 1

Clinical Characteristics

\begin{tabular}{|c|c|c|c|}
\hline Characteristics & $\begin{array}{l}\text { All patients } \\
(n=126)\end{array}$ & $\begin{array}{c}\mathrm{i}-\mathrm{PET} \\
(n=100)\end{array}$ & $\begin{array}{l}\text { EoT-PET } \\
(n=95)\end{array}$ \\
\hline \multicolumn{4}{|l|}{ Age } \\
\hline Median (y) & 64.6 & 63.6 & 63.0 \\
\hline Range (y) & $20-88$ & $20-78$ & $20-88$ \\
\hline$\leq 60 \mathrm{y}$ & $52(41.3)$ & $44(44.0)$ & $43(45.3)$ \\
\hline $60 \mathrm{y}$ & $74(58.7)$ & $56(56.0)$ & $52(54.7)$ \\
\hline \multicolumn{4}{|l|}{ Sex } \\
\hline Female & $56(44.4)$ & $47(47.0)$ & $44(46.3)$ \\
\hline Male & $70(55.6)$ & $53(53.0)$ & $51(53.7)$ \\
\hline \multicolumn{4}{|l|}{ Body mass index } \\
\hline Mean $\pm \mathrm{SD}\left(\mathrm{kg} / \mathrm{m}^{2}\right)$ & $26.4 \pm 5.5$ & $26.3 \pm 5.7$ & $26.5 \pm 5.7$ \\
\hline$\leq 25 \mathrm{~kg} / \mathrm{m}^{2}$ & $57(45.2)$ & 49 (49.0) & $42(44.2)$ \\
\hline $25 \mathrm{~kg} / \mathrm{m}^{2}$ & $69(54.8)$ & $51(51.0)$ & $53(55.8)$ \\
\hline \multicolumn{4}{|l|}{$\begin{array}{l}\text { ECOG performance } \\
\text { status }\end{array}$} \\
\hline 0 & $43(34.1)$ & $38(38.0)$ & $29(30.5)$ \\
\hline 1 & $50(39.7)$ & $36(36.0)$ & $38(40.0)$ \\
\hline 2 & 27 (21.4) & $22(22.0)$ & $23(24.2)$ \\
\hline 3 & $6(4.8)$ & $4(4.0)$ & $5(5.3)$ \\
\hline 4 & $0(0)$ & $0(0)$ & $0(0)$ \\
\hline \multicolumn{4}{|c|}{ Lactate dehydrogenase } \\
\hline$\leq$ normal & $54(42.9)$ & $55(55.0)$ & $37(38.9)$ \\
\hline normal & $72(57.1)$ & $45(45.0)$ & $58(61.1)$ \\
\hline \multicolumn{4}{|l|}{ Ann Arbor stage } \\
\hline I & $16(12.7)$ & $14(14.0)$ & $13(13.7)$ \\
\hline ॥ & $26(20.6)$ & $23(23.0)$ & $16(16.8)$ \\
\hline III & $26(20.6)$ & $20(20.0)$ & $20(21.1)$ \\
\hline IV & $58(46.0)$ & $43(43.0)$ & $46(48.4)$ \\
\hline \multicolumn{4}{|c|}{ Extranodal involvement } \\
\hline 0 or 1 & $97(77.0)$ & $80(80.0)$ & $73(76.8)$ \\
\hline$\geq 2$ & $29(23.0)$ & $20(20.0)$ & $22(23.2)$ \\
\hline \multicolumn{4}{|l|}{$\begin{array}{l}\text { Bone marrow } \\
\text { involvement }\end{array}$} \\
\hline Yes & $9(7.1)$ & $8(8.0)$ & $7(7.4)$ \\
\hline No & $76(60.3)$ & $63(63.0)$ & $58(61.0)$ \\
\hline Not applicable & $41(32.6)$ & $29(29.0)$ & $30(31.6)$ \\
\hline \multicolumn{4}{|l|}{ Age-adjusted IPI } \\
\hline 0 & $25(19.8)$ & $22(22.0)$ & $14(14.7)$ \\
\hline 1 & 38 (30.2) & $32(32.0)$ & $30(31.6)$ \\
\hline 2 & 38 (30.2) & $26(26.0)$ & $31(32.6)$ \\
\hline 3 & 25 (19.8) & $20(20.0)$ & $20(21.1)$ \\
\hline \multicolumn{4}{|l|}{ B symptoms } \\
\hline Presence & 23 (18.3) & 17 (17.0) & 74 (77.9) \\
\hline Absence & $103(81.7)$ & $83(83.0)$ & 21 (22.1) \\
\hline \multicolumn{4}{|l|}{$\begin{array}{l}\text { First line of } \\
\text { chemotherapy }\end{array}$} \\
\hline $\mathrm{RCHOP}$ & $108(85.7)$ & $83(83.0)$ & 79 (77.9) \\
\hline RACVBP & $18(14.3)$ & 17 (17.0) & $21(22.1)$ \\
\hline \multicolumn{4}{|l|}{ Bulky disease } \\
\hline Yes & 44 (34.9) & $34(34.0)$ & 39 (41.1) \\
\hline No & $82(65.1)$ & $66(66.0)$ & $56(58.9)$ \\
\hline
\end{tabular}

ECOG = Eastern Cooperative Oncology Group; IPI = international prognostic index; $\mathrm{RCHOP}=$ rituximab, cyclophosphamide, doxorubicin, vincristine, prednisone; RACVBP

$=$ rituximab, doxorubicin, cyclophosphamide, vindesine, bleomycin, prednisone.

Data are $n$ followed by percentage in parentheses, unless indicated otherwise. number of patients compared with a reconstruction meeting the EANM/EARL harmonizing standards. The clinical relevance of induced changes was assessed by studying progression-free survival (PFS) and overall survival (OS) in responders versus nonresponders to determine whether potential discordance would affect the risk stratification capability of ${ }^{18}$ F-FDG PET in lymphoma patients.

\section{MATERIALS AND METHODS}

\section{Patient Selection}

Patients newly diagnosed with diffuse large B-cell lymphoma (DLBCL) between October 2008 and September 2015 were included in this retrospective study. The patients were referred to our PET unit for baseline PET, i-PET after 4 courses of chemotherapy, or EoT-PET.

For each patient, baseline data were recorded, including age, sex, body mass index, ECOG performance status, lactate dehydrogenase rate, Ann Arbor stage, extranodal involvement, presence or absence of bone marrow involvement, age-adjusted international prognosis index, presence or absence of B symptoms, and presence or absence of bulky disease.

All patients were treated with standard chemotherapy depending on the stage, age, and site of initial involvement. Either the patients received rituximab, cyclophosphamide, doxorubicin, vincristine, and prednisone, or they received rituximab, doxorubicin, cyclophosphamide, vindesine, bleomycin, and prednisone. Follow-up data were recorded at scheduled visits.

According to European regulations, French observational studies without any additional therapy or monitoring procedures do not need ethics approval. Nonetheless, approval to collect data for our study was obtained from the national committee for data privacy (registration no. $2084622 \mathrm{v} 0)$.

\section{PET Acquisition and Reconstruction Parameters}

PET studies were performed per the EANM guidelines for PET tumor imaging (12). Patients fasted at least $6 \mathrm{~h}$ before receiving an intravenous injection of ${ }^{18} \mathrm{~F}$-FDG (mean injected dose $\pm \mathrm{SD}, 4.00 \pm$ $0.22 \mathrm{MBq} / \mathrm{kg}$ ). The mean blood glucose level was $1.03 \pm 0.23 \mathrm{~g} / \mathrm{L}$ at the time of injection. According to the EANM guidelines, all PET studies were performed after $60 \pm 5 \mathrm{~min}$ of rest in a warm room (mean delay between injection and acquisition, $61.4 \pm 6.5 \mathrm{~min}$ ) on a Biograph TrueV system (Siemens Medical Solutions) with a 6-slice spiral CT component.

First, a free-breathing $\mathrm{CT}$ acquisition was performed using the following parameters: $60 \mathrm{mAs}, 130 \mathrm{kVp}$, pitch of 1 , and $6 \times 2 \mathrm{~mm}$ collimation. Then, the PET emission acquisition was performed in 3dimensional mode. Patients were scanned from the base of their skull to their mid thighs, with acquisition times per bed position of $160 \mathrm{~s}$ for normal-weight patients (body mass index $\leq 25 \mathrm{~kg} / \mathrm{m}^{2}$ ) and $220 \mathrm{~s}$ for overweight patients (body mass index $>25 \mathrm{~kg} / \mathrm{m}^{2}$ ), respectively.

Raw data were reconstructed with a PSF reconstruction algorithm (HD.PET data reconstructed with TrueX algorithm; Siemens Medical Solutions) with 3 iterations and 21 subsets and no filtering. The matrix size was $168 \times 168$ voxels, which resulted in voxels of $4.07 \times 4.07 \times 4.07 \mathrm{~mm}$. Scatter and CT attenuation correction based on the CT scan was applied.

\section{PET Analysis}

PET analysis was performed using Syngo.via and EQ.PET software (Siemens Medical Solutions). EQ.PET computes SUV on images optimized for diagnosis (in our center, unfiltered PSF) while having access to SUVs to meet the EANM/EARL harmonizing standards with no need for a second reconstruction dataset (13). EQ.PET has been shown to provide SUV metrics that are consistent with those produced by a second reconstruction (14). 
TABLE 2

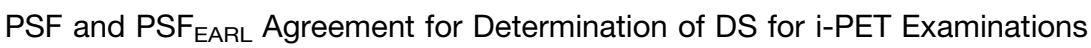

\begin{tabular}{|c|c|c|c|c|c|c|}
\hline \multirow[b]{2}{*}{$\operatorname{PSF}_{\text {EARL }}(n)$} & \multicolumn{5}{|c|}{$\operatorname{PSF}(n)$} & \multirow[b]{2}{*}{ Total } \\
\hline & 1 & 2 & 3 & 4 & 5 & \\
\hline 1 & 24 & 0 & 0 & 0 & 0 & 24 (24.0\%) \\
\hline 2 & 0 & 12 & 5 & 0 & 0 & 17 (17.0\%) \\
\hline 3 & 0 & 1 & 17 & 4 & 0 & 22 (22.0\%) \\
\hline 4 & 0 & 0 & 1 & 27 & 3 & 31 (31.0\%) \\
\hline 5 & 0 & 0 & 0 & 0 & 6 & $6(6.0 \%)$ \\
\hline Total & $24(24.0 \%)$ & $13(13.0 \%)$ & $23(23.0 \%)$ & 31 (31.0\%) & $9(9.0 \%)$ & $100(100 \%)$ \\
\hline
\end{tabular}

TABLE 3

Characteristics of Patients Presenting with Major Discordance (DS Moving from DS3 to DS4 and Vice Versa) Between PSF and PSF EARL

\begin{tabular}{|c|c|c|c|c|c|c|c|c|}
\hline \multirow[b]{2}{*}{ Parameter } & \multicolumn{5}{|c|}{ i-PET } & \multicolumn{3}{|c|}{ EOT-PET } \\
\hline & Patient 1 & Patient 2 & Patient 3 & Patient 4 & Patient 5 & Patient 6 & Patient 7 & Patient 8 \\
\hline \multicolumn{9}{|l|}{ Characteristics } \\
\hline Age (y) & 59 & 62 & 64 & 65 & 63 & 81 & 71 & 71 \\
\hline Sex & $\mathrm{F}$ & $\mathrm{F}$ & $\mathrm{F}$ & $\mathrm{M}$ & $\mathrm{F}$ & $\mathrm{F}$ & $\mathrm{M}$ & $\mathrm{M}$ \\
\hline Body mass index $\left(\mathrm{kg} / \mathrm{m}^{2}\right)$ & 31.2 & 30.8 & 22.1 & 28.7 & 38.1 & 28.5 & 33.8 & 26.7 \\
\hline Ann Arbor stage & IV & III & IV & III & II & IV & III & IV \\
\hline $\mathrm{LDH}$ & $>$ Normal & $\leq$ Normal & $>$ Normal & $\leq$ Normal & $\leq$ Normal & $>$ Normal & $>$ Normal & $>$ Normal \\
\hline $\begin{array}{l}\text { ECOG Performance } \\
\text { status }\end{array}$ & 2 & 0 & 2 & 0 & 0 & 2 & 2 & 2 \\
\hline Extranodal involvement & 1 & 0 & 4 & 0 & 0 & 2 & 3 & 1 \\
\hline Age-adjusted IPI & 3 & 1 & 3 & 1 & 0 & 3 & 3 & 3 \\
\hline Bulky disease & No & No & No & No & No & Yes & Yes & No \\
\hline \multicolumn{9}{|l|}{ Follow-up } \\
\hline Progression & No & No & $\begin{array}{c}\text { Yes (5-mo } \\
\text { delay) }\end{array}$ & No & No & $\begin{array}{l}\text { Yes (16-mo } \\
\text { delay) }\end{array}$ & No & No \\
\hline $\begin{array}{l}\text { Location of } \\
\text { progression }\end{array}$ & & & Meningeal & & & $\begin{array}{l}\text { Right axillary } \\
\text { node }\end{array}$ & & \\
\hline Death (delay) & No & No & $\begin{array}{c}\text { Yes (9-mo } \\
\text { delay) }\end{array}$ & No & No & No & No & No \\
\hline \multicolumn{9}{|l|}{ PET data } \\
\hline $\begin{array}{l}\text { Location of initial } \\
\text { mass }\end{array}$ & $\begin{array}{l}\text { Anterior renal } \\
\text { fascia }\end{array}$ & $\begin{array}{l}\text { Mesenteric } \\
\text { mass }\end{array}$ & $\begin{array}{l}\text { L external } \\
\text { iliac node }\end{array}$ & $\begin{array}{l}\text { R cervical } \\
\text { node }\end{array}$ & $\begin{array}{l}\text { Supraclavicular } \\
\text { node }\end{array}$ & $\begin{array}{l}\mathrm{R} \text { inguinal } \\
\text { node }\end{array}$ & $\begin{array}{l}\text { Mesenteric } \\
\text { mass }\end{array}$ & $\begin{array}{l}\text { L cervical } \\
\text { node }\end{array}$ \\
\hline $\begin{array}{l}\text { Initial SUV lesion } \\
\text { PSF/PSF } \\
\text { EARL }\end{array}$ & $37.93 / 29.39$ & $31.39 / 25.64$ & $18.42 / 15.54$ & $16.40 / 12.94$ & $15.15 / 9.85$ & $27.12 / 18.02$ & $30.87 / 24.82$ & $48.53 / 31.66$ \\
\hline $\begin{array}{l}\text { Location of residual } \\
\text { mass }\end{array}$ & L pleura & $\begin{array}{l}\text { Mesenteric } \\
\text { node }\end{array}$ & Pericardium & $\begin{array}{l}\text { R cervical } \\
\text { node }\end{array}$ & $\begin{array}{l}\text { Anterior } \\
\text { mediastinum }\end{array}$ & $\begin{array}{l}\text { R axillary } \\
\text { node }\end{array}$ & $\begin{array}{l}\text { Mesenteric } \\
\text { mass }\end{array}$ & Celiac node \\
\hline $\begin{array}{l}\text { Size of residual mass } \\
\quad(\mathrm{mm})\end{array}$ & 27 & 11 & 7 & 20 & 21 & 6 & 62 & 20 \\
\hline SUV $_{\text {lesion }}$ PSF/PSF EARL & $4.61 / 3.26$ & $4.21 / 3.29$ & $4.01 / 2.79$ & $4.36 / 2.86$ & $4.82 / 3.85$ & $3.93 / 2.49$ & $4.12 / 2.90$ & $3.31 / 2.66$ \\
\hline SUV $_{\text {liver }}$ PSF/PSF $F_{\text {EARL }}$ & $4.02 / 3.35$ & $3.96 / 3.45$ & $3.59 / 3.12$ & $4.23 / 3.32$ & $4.88 / 3.58$ & $3.58 / 3.04$ & $3.85 / 3.22$ & $3.29 / 2.75$ \\
\hline $\begin{array}{l}\text { SUV }_{\text {mediastinum }} \\
\quad \text { PSF/PSF } \\
\text { EARL }\end{array}$ & $2.71 / 2.21$ & $3.08 / 2.69$ & $2.66 / 2.11$ & $2.86 / 2.31$ & $3.12 / 2.63$ & $2.27 / 2.07$ & $2.46 / 2.13$ & $2.22 / 1.85$ \\
\hline DS-status PSF & $4, N R$ & $4, N R$ & $4, N R$ & $4, N R$ & $3, \mathrm{R}$ & $4, N R$ & $4, N R$ & $4, N R$ \\
\hline DS-status PSF EARL & $3, \mathrm{R}$ & $3, \mathrm{R}$ & $3, \mathrm{R}$ & $3, \mathrm{R}$ & $4, \mathrm{NR}$ & $3, \mathrm{R}$ & $3, \mathrm{R}$ & $3, \mathrm{R}$ \\
\hline PSF $\Delta$ SUV $_{\max }$ & 87.8 & 86.6 & 78.2 & 73.4 & 68.2 & & & \\
\hline $\mathrm{PSF}_{\text {EARL }} \Delta \mathrm{SUV}_{\max }$ & 88.9 & 87.2 & 82.0 & 77.9 & 60.9 & & & \\
\hline
\end{tabular}

$\mathrm{LDH}=$ lactate dehydrogenase; ECOG = Eastern Cooperative Oncology Group; IPI= international prognostic index; $\mathrm{R}=$ responder; NR = nonresponder. 
TABLE 4

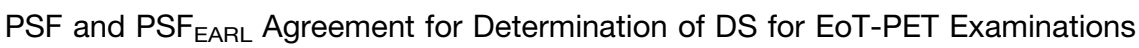

\begin{tabular}{lcccccc}
\hline & \multicolumn{5}{c}{ PSF $(n)$} & \multirow{2}{*}{ Total } \\
\cline { 2 - 5 } PSF $_{\text {EARL }}(n)$ & 1 & 2 & 3 & 4 & 5 & $36(37.9 \%)$ \\
\hline 1 & 36 & 0 & 0 & 0 & 0 & $18(18.9 \%)$ \\
2 & 0 & 14 & 4 & 0 & 0 & $18(18.9 \%)$ \\
3 & 0 & 0 & 15 & 3 & 0 & $15(15.8 \%)$ \\
4 & 0 & 0 & 0 & 14 & 1 & $8(8.4 \%)$ \\
5 & 0 & 0 & 0 & 0 & 8 & $95(100 \%)$ \\
\hline
\end{tabular}

All PET examinations were reviewed by a single board-certified PET reader using PSF images for optimal lesion detection. Subsequently, all quantitative values were recorded after application of a 6-mm gaussian filter to obtain harmonized SUV according to the EARL harmonization program ( $\mathrm{PSF}_{\mathrm{EARL}}$ ).

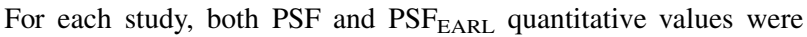
recorded. Liver $\mathrm{SUV}_{\max }\left(\mathrm{SUV}_{\text {liver }}\right)$ was automatically measured using a 3-cm-diameter volume of interest placed in the right liver lobe. In cases of focal liver involvement, liver lesions were avoided, and in cases of diffuse liver involvement, examinations were excluded. Mediastinum $\mathrm{SUV}_{\max }\left(\mathrm{SUV}_{\text {mediastinum }}\right)$ was automatically measured using a 1-cm-diameter and a 2-cm-high cylinder in the descending aorta. Lesion $\mathrm{SUV}_{\max }\left(\mathrm{SUV}_{\text {lesion }}\right)$ was measured with a manual volume of interest placed in the most intense residual target lesions. For this purpose, the baseline scan was systematically reviewed to make sure this site was initially involved.

i-PET and EoT-PET results were scored according to the DS (4) as DS1 (no residual uptake), DS2 (uptake $\leq$ mediastinum), DS3 (uptake $>$ mediastinum but $\leq$ liver), DS4 (moderately increased uptake $>$ liver), or DS5 (markedly increased uptake $>$ liver, defined as $\mathrm{SUV}_{\text {lesion }} \geq 2 \times$ $\mathrm{SUV}_{\text {liver }}$ ) or new lesions related to lymphoma.

Patients with DS1-DS3 were classified as responders. Patients with DS4 or DS5 were classified as nonresponders. $\Delta \mathrm{SUV}_{\max }$ was computed as follows:

$$
\Delta \mathrm{SUV}_{\max }=\frac{\text { baseline } \mathrm{SUV}_{\text {lesion }}-\text { interim } \mathrm{SUV}_{\text {lesion }}}{\text { baseline } \mathrm{SUV}_{\text {lesion }}} \times 100 .
$$

The cutoff to be classified as responder was $\Delta \mathrm{SUV}_{\max }$ greater than $70 \%$ (15).

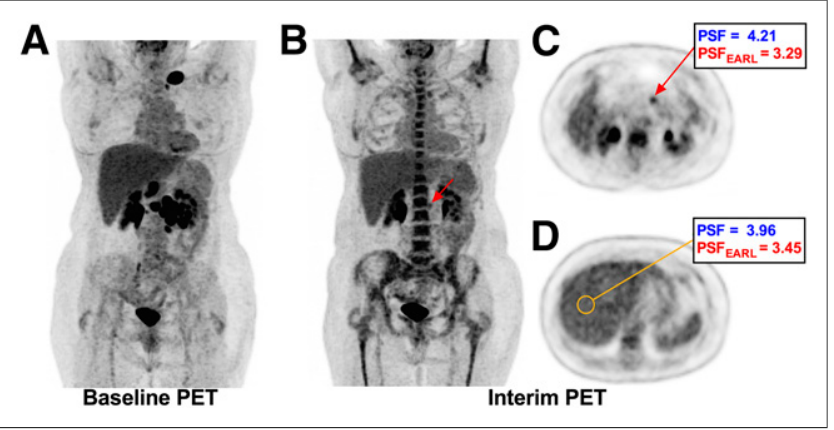

FIGURE 1. Representative example of patient who had major discordance between PSF and PSF EARL $_{\text {DSs. This patient was classified as }}$

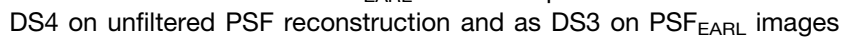
(patient 2 in Table 3). Maximum-intensity projection view for baseline PET (A), i-PET (B), and transverse slices at level of lesion (C) and automatic liver VOI (D) are shown.

\section{Statistical Analysis}

Quantitative data are presented as the mean \pm SD or, when needed, as the median and range. Agreement between PSF and PSF EARL $_{\text {re- }}$ constructions was assessed by calculating the Cohen unweighted $\kappa$. The prognostic value of i-PET and EoT-PET was assessed using PFS and OS. PFS was defined as the time from diagnosis to progression or death, and OS was the time from diagnosis to death from the lymphoma disease (lymphoma itself or treatment side effects). Survival curves were obtained with the Kaplan-Meier test and compared with the log-rank test. Statistical significance was considered present at a $P$ value of less than 0.05 . Graphs and analyses were produced using GraphPad Prism and MedCalc software.

\section{RESULTS}

\section{Patient Characteristics}

A database of 195 PET examinations (100 i-PET and 95 EoTPET) for 126 patients was created. Patient clinical characteristics are displayed in Table 1. Thirty-one patients underwent only iPET, 26 patients underwent only EoT-PET, and 69 patients underwent both i-PET and EoT-PET. Diffuse liver involvement did not occur, and therefore no examination was excluded.

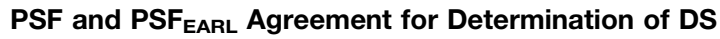

I-PET Examinations. For i-PET examinations, patients were classified using PSF SUVs as DS1, DS2, DS3, DS4, and DS5 in $24,13,23,31$, and 9 cases, respectively. These patients were

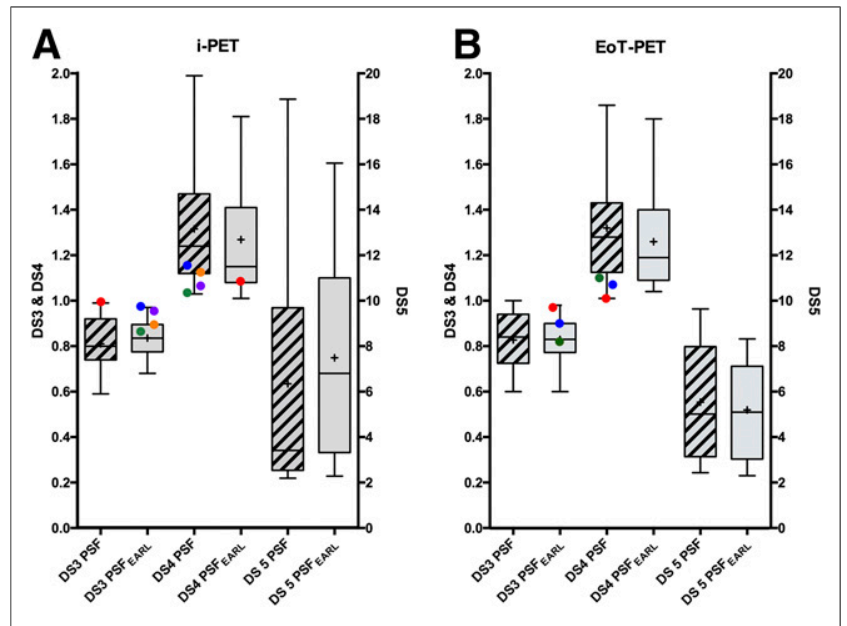

FIGURE 2. Liver-to-lesion ratio for patients scored DS3-DS5. Lines denote median and interquartile ranges. Paired colored points correspond to discordance between unfiltered PSF and PSF EARL $_{\text {. }}$ 


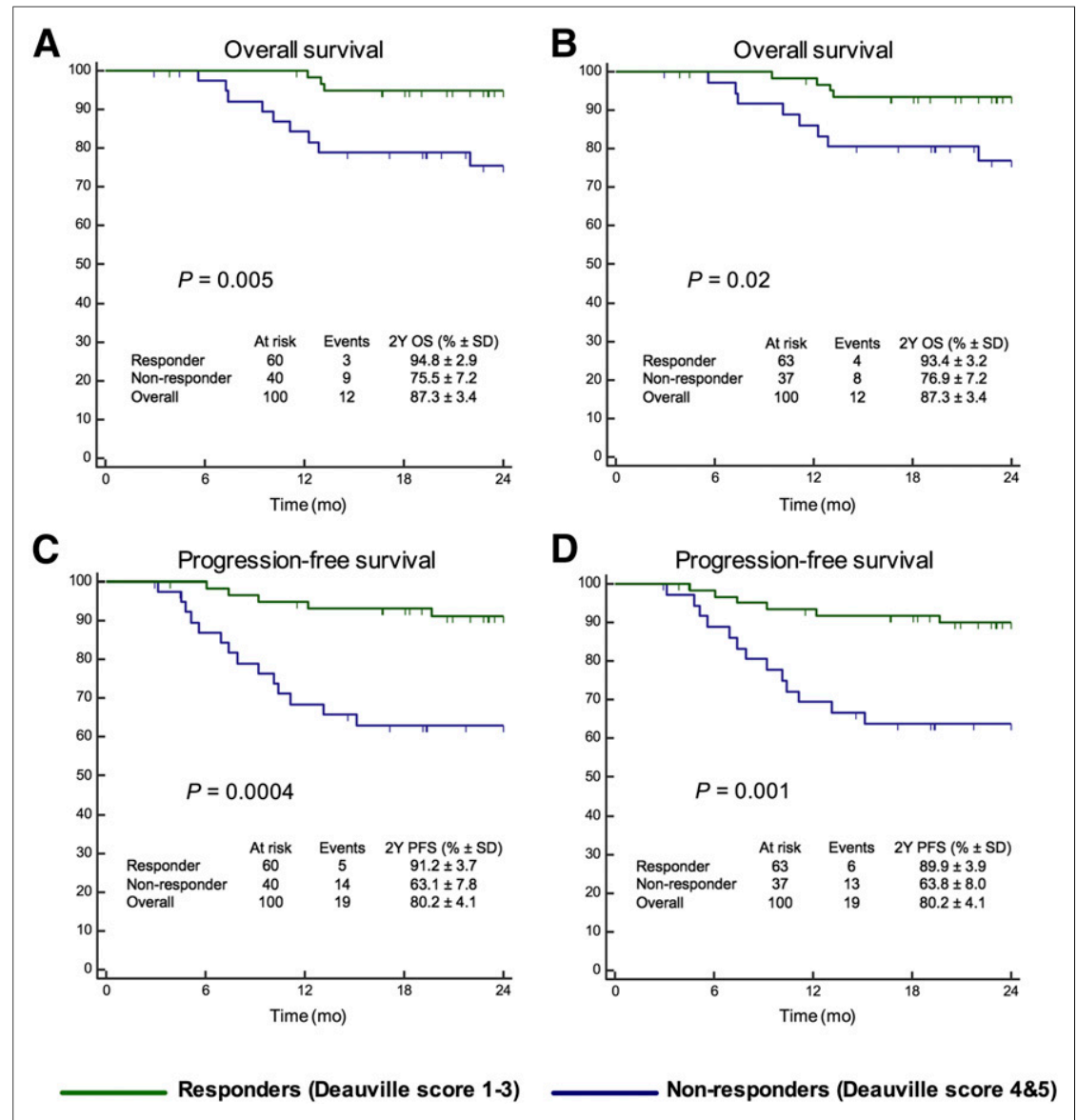

FIGURE 3. PFS and OS analysis among responder patients (DS1-DS3) and nonresponder patients (DS4 and DS5) on i-PET. Data are shown for unfiltered PSF (A and C) and for PSF EARL (B and D).

classified as 60 responders and 40 nonresponders. Patients were

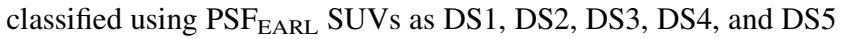
in $24,17,22,31$, and 6 cases, respectively, which resulted in 63 responders and 37 nonresponders. DS discordance between PSF and $\mathrm{PSF}_{\mathrm{EARL}}$ occurred in 14 cases (14.0\%): 1 patient moved from DS2 to DS3, 5 from DS3 to DS2, 1 from DS3 to DS4, 4 from DS4 to DS3, and 3 from DS5 to DS4 (Table 2). Concordance between

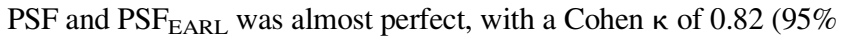
confidence interval, 0.73-0.91). Noticeably, only 5 cases of major discordance (5.0\%) occurred: 4 patients classified as nonresponders with PSF became responders with PSF $_{\text {EARL }}$, and 1 patient classified as a responder with PSF became a nonresponder with PSF EARL. $_{\text {. }}$

Table 3 presents the clinical and PET characteristics, as well as the outcomes, for these patients. When focusing on the $\Delta \mathrm{SUV}_{\max }$ of these 5 patients (Table 3), we found that PSF and $\mathrm{PSF}_{\text {EARL }} \Delta \mathrm{SUV}_{\max }$ status was concordant in all cases. Interestingly, the $\Delta \mathrm{SUV}_{\max }$ status always matched the PSF $\mathrm{EARL}_{\mathrm{ES}}$ DS status. Also, an excellent correlation was found between $\triangle \mathrm{SUV}_{\max }$ for all the patients of the i-PET group as shown in Supplemental Figure 1 (supplemental materials are available at http://jnm.snmjournals.org).

EoT-PET Examinations. For EoT-PET examinations, patients were classified using PSF SUVs as DS1, DS2, DS3, DS4, and DS5 in $36,14,19,17$, and 9 cases, respectively. This result led to 69 responders and 26 nonresponders. Patients were classified using PSF $_{\text {EARL }}$ SUVs as DS1, DS2, DS3, DS4, and DS5 in 36, 18, 18, 15, and 8 cases, respectively, which resulted in 72 responders and 23 nonresponders. Eight discordant cases occurred $(8.4 \%)$ between

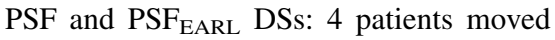
from DS3 to DS2, 3 from DS4 to DS3, and 1 from DS5 to DS4 (Table 4). Concordance

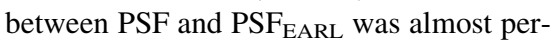
fect, with a $\kappa$ of 0.89 (95\% confidence interval, 0.81-0.96). Notably, only 3 cases of major discordance occurred among the patients $(3.2 \%)$, with these involving a change from nonresponders according to PSF to responders according to $\mathrm{PSF}_{\mathrm{EARL}}$.

Table 3 presents the clinical and PET characteristics, as well as the outcomes, for these patients. Figure 1 displays a representative example of a patient who had major discordance between PSF and PSF EARL DSs. Figure 2 displays the lesion-to-liver ratios in DS3-DS5 patients.

\section{Survival Analysis}

I-PET Examinations. For i-PET examinations, the median follow-up time for patients was 28.4 mo (range, 3-84 mo). At 2 y, 15 patients $(15.0 \%)$ experienced progression or relapse of their DLBCL, and 12 patients $(12.0 \%)$ died from lymphoma disease (lymphoma itself or treatment side effects). For the whole group, the estimated PFS at 2 y was $80.2 \% \pm 4.1 \%$, and the estimated OS at $2 \mathrm{y}$ was $87.3 \% \pm 3.4 \%$. When PSF was used to classify patients, there was a significant difference between the OS and PFS of responders versus nonresponders (Figs. 3A and 3C). The same degree of significance was observed with PSF EARL $_{\text {(Figs. 3B }}$ and 3D).

EoT-PET Examinations. For EoT-PET examinations, the median follow-up time for patients was 27.4 mo (range, 6-84 mo). At 2 y, 15 patients $(15.8 \%)$ experienced progression or relapse of their DLBCL, and 10 patients $(10.5 \%)$ died from the lymphoma disease (either from lymphoma itself or treatment side effects). For the whole group, the estimated PFS at 2 y was $80.3 \% \pm$ $4.2 \%$, and the estimated OS at 2 y was $88.5 \% \pm 3.4 \%$. When PSF was used to classify patients, there was a significant difference between the OS and PFS of responders versus nonresponders (Figs. 4A and 4C). Similar results were observed with PSF $F_{\text {EARL }}$ (Figs. 4B and 4D).

\section{DISCUSSION}

In this study, using a methodology to avoid any inter- or intraobserver variability, the proportion of discordant cases in DS when reconstructing PET raw data with either unfiltered PSF reconstruction or EARL-compliant reconstruction was moderate $(14.0 \%$ in i-PET and $8.4 \%$ in EoT-PET). When classifying patients as responders (DS1-DS3) versus nonresponders (DS4 and DS5), the frequency of discordance was even lower: $5.0 \%$ in i-PET and $3.2 \%$ in EoT-PET. More important, we assessed the clinical relevance of changes induced by the use of PSF modeling and found that the risk stratification capability of ${ }^{18}$ F-FDG PET in DLBCL patients 


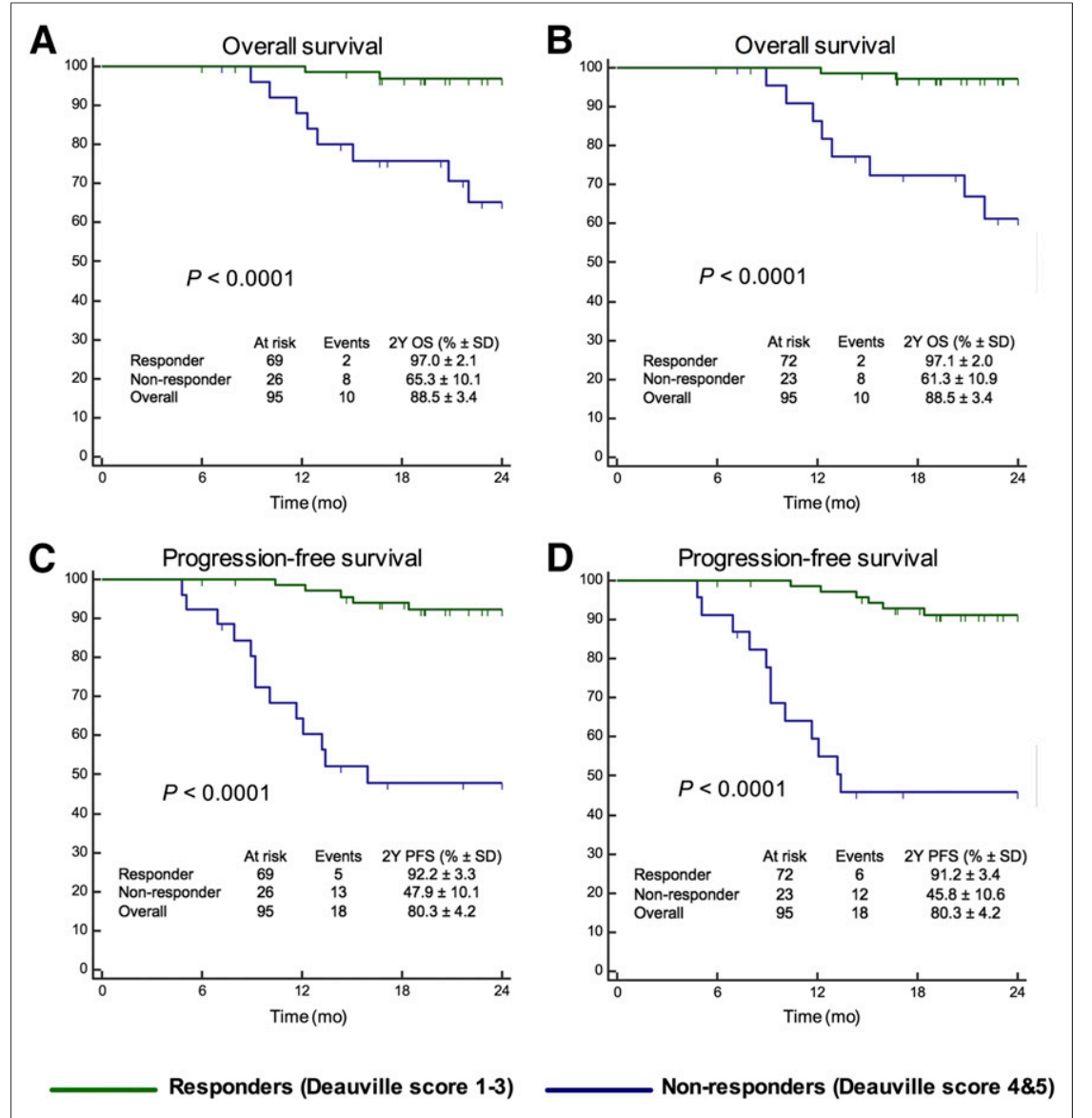

FIGURE 4. PFS and OS analysis among responder patients (DS1-DS3) and nonresponder patients (DS4 and DS5) for EoT-PET. Data are shown for unfiltered PSF (A and C) and for PSF EARL (B and D).

was not affected by the choice of the reconstruction algorithm because similar 2-y PSF and OS were observed for both algorithms. Of note, most of the discordant cases were related to minimal changes in lesion-to-liver ratio on PSF versus EARL-compliant images and would have been eliminated by using a less stringent cutoff, such as a 1.4 lesion-to-liver ratio for i-PET (10).

It is noteworthy that the PSF reconstruction setting used in our PET center can be considered the worst-case scenario in terms of SUV reconstruction dependency because we do not use any postfiltering. This approach leads to the highest differences in SUV in residual masses compared with a standard algorithm, such as ordered-subset expectation maximization, or an EARLcompliant reconstruction. Our group has reported an increase in $\mathrm{SUV}_{\max }$ equal to $66 \%$ for unfiltered PSF compared with an ordered-subset expectation maximization reconstruction meeting the EARL requirements for small nodal metastases (7). Many centers tend to use a filter with a small kernel (2-3 mm) (13), and therefore it is likely that the number of discordant cases in DS would have been even lower in these centers.

On the basis of the present results, one could conclude that, unlike what was cautiously recommended by some multicenter studies such as the RATHL trial (10), disabling the PSF or PSF+TOF capability of a PET system seems not to be warranted when obtaining DSs for a PET response-adapted trial. Furthermore, because PSF and PSF+TOF quantification capabilities have been shown to be almost similar in terms of activity recovery (13), this statement could be extrapolated to PSF+TOF. Going further, and keeping in mind that quantitative data from a baseline scan are not required to obtain DSs on i-PET or EoT-PET (only visualization of the baseline scan is still highly recommended to identify the initial locations of the disease and select the target lesion for a DS assessment, which results in better interobserver concordance (17)), one could consider that a patient could in fact be scanned on different PET systems at baseline and follow-up.

However, several points mitigate the use of PSF in PET-driven trials and tolerance of reconstruction inconsistencies between baseline and posttreatment scans. First, scanning a patient on different PET systems would be an issue when computing $\triangle$ SUV between baseline scan and i-PET (18). For example, the use of an EARLcompliant system for baseline, along with an advanced algorithm, such as PSF for i-PET, would artificially lower the $\triangle$ SUV and provide an inaccurate therapeutic assessment, which could potentially lead to inadequate changes in patient case management. Additionally, the use of SUV metrics as prognostic factors when pooling data from several PET systems would be affected by reconstruction inconsistencies. Finally, the delineation of metabolically active tumor volume, which is increasingly being used as a prognosticator in lymphoma patients $(19,20)$, is strongly affected by the use of advanced reconstruction algorithms (21). For these reasons, it seems preferable to pursue ongoing harmonization efforts with programs such as EARL or QIBA.

In favor of the harmonized reconstructions, it is noteworthy that analysis of discordant cases in the i-PET group (Table 3) showed that $\triangle \mathrm{SUV}$ in those cases was always concordant between both algorithms (i.e., above or below the $70 \%$ validated threshold after 4 cycles of chemotherapy in DLBCL patients (18)) and was also concordant with the DS provided by the EARL-compliant reconstruction. This finding has to be confirmed, of course, by further studies investigating the impact of the reconstruction algorithm on $\Delta$ SUV.

Finally, although this was not investigated in the present study, it is noteworthy that discordance between PSF and EARLcompliant reconstructions led to changes from DS2 to DS3 that would affect the outcome of deescalation trials. Indeed, in those trials, DS3 on early i-PET is generally not considered to be sufficient to allow entry into the deescalated arm, and DS2 is required $(1,5)$. Further research is therefore needed before the findings of the present study are applied to deescalation trials.

\section{CONCLUSION}

In this study, the i-PET and EoT-PET DSs of DLBCL patients were minimally affected by the choice of PET reconstruction method, and the observed changes did not affect the risk stratification capability of ${ }^{18}$ F-FDG PET. The use of advanced 
reconstruction algorithms, such as PSF modeling, seems not to be an issue in routine clinical procedures or in multicenter trials when the DS is computed. However, it seems preferable to pursue ongoing harmonization efforts, at least when computing $\triangle \mathrm{SUV}$ or pooling SUVs provided by different PET systems.

\section{DISCLOSURE}

No potential conflict of interest relevant to this article was reported.

\section{REFERENCES}

1. Barrington SF, Kluge R. FDG PET for therapy monitoring in Hodgkin and nonHodgkin lymphomas. Eur J Nucl Med Mol Imaging. 2017;44:97-110.

2. Barrington SF, Qian W, Somer EJ, et al. Concordance between four European centres of PET reporting criteria designed for use in multicentre trials in Hodgkin lymphoma. Eur J Nucl Med Mol Imaging. 2010;37:1824-1833.

3. Biggi A, Gallamini A, Chauvie S, et al. International validation study for interim PET in ABVD-treated, advanced-stage Hodgkin lymphoma: interpretation criteria and concordance rate among reviewers. $J$ Nucl Med. 2013;54:683-690.

4. Meignan M, Gallamini A, Haioun C, et al. Report on the 5th International Workshop on Positron Emission Tomography in Lymphoma held in Menton, France, 19-20 September 2014. Leuk Lymphoma. 2015;56:1229-1232.

5. Cheson BD, Fisher RI, Barrington SF, et al. Recommendations for initial evaluation, staging, and response assessment of Hodgkin and non-Hodgkin lymphoma: the Lugano classification. J Clin Oncol. 2014;32:3059-3068.

6. van der Vos CS, Koopman D, Rijnsdorp S, et al. Quantification, improvement, and harmonization of small lesion detection with state-of-the-art PET. Eur J Nucl Med Mol Imaging. 2017;44:4-16.

7. Bellevre D, Blanc Fournier C, Switsers O, et al. Staging the axilla in breast cancer patients with ${ }^{18} \mathrm{~F}-\mathrm{FDG}$ PET: how small are the metastases that we can detect with new generation clinical PET systems? Eur J Nucl Med Mol Imaging. 2014;41:1103-1112.

8. Kuhnert G, Boellaard R, Sterzer S, et al. Impact of PET/CT image reconstruction methods and liver uptake normalization strategies on quantitative image analysis. Eur J Nucl Med Mol Imaging. 2016;43:249-258.
9. Quak E, Hovhannisyan N, Lasnon C, et al. The importance of harmonizing interim positron emission tomography in non-Hodgkin lymphoma: focus on the Deauville criteria. Haematologica. 2014;99:e84-e85.

10. Barrington SF, Kirkwood AA, Franceschetto A, et al. PET-CT for staging and early response: results from the Response-Adapted Therapy in Advanced Hodgkin Lymphoma Study. Blood. 2016;127:1531-1538.

11. Aide N, Lasnon C, Veit-Haibach P, Sera T, Sattler B, Boellaard R. EANM/EARL harmonization strategies in PET quantification: from daily practice to multicentre oncological studies. Eur J Nucl Med Mol Imaging. 2017;44:17-31.

12. Boellaard R, Delgado-Bolton R, Oyen WJ, et al. FDG PET/CT: EANM procedure guidelines for tumour imaging: version 2.0. Eur J Nucl Med Mol Imaging. 2015;42:328-354.

13. Quak E, Le Roux PY, Hofman MS, et al. Harmonizing FDG PET quantification while maintaining optimal lesion detection: prospective multicentre validation in 517 oncology patients. Eur J Nucl Med Mol Imaging. 2015;42: 2072-2082.

14. Lasnon C, Salomon T, Desmonts C, et al. Generating harmonized SUV within the EANM EARL accreditation program: software approach versus EARL-compliant reconstruction. Ann Nucl Med. 2017;31:125-134.

15. Casasnovas RO, Meignan M, Berriolo-Riedinger A, et al. SUVmax reduction improves early prognosis value of interim positron emission tomography scans in diffuse large B-cell lymphoma. Blood. 2011;118:37-43.

16. Zhang Y, Fan Y, Ying Z, et al. Can the SUVmax-liver-based interpretation improve prognostic accuracy of interim and posttreatment ${ }^{18} \mathrm{~F}$-FDG PET/CT in patients with diffuse large B-cell lymphoma? Leuk Lymphoma. 2018;59:660-669.

17. Quarles van Ufford $\mathrm{H}$, Hoekstra $\mathrm{O}$, de Haas $\mathrm{M}$, et al. On the added value of baseline FDG-PET in malignant lymphoma. Mol Imaging Biol. 2010;12:225-232.

18. Itti E, Lin C, Dupuis J, et al. Prognostic value of interim ${ }^{18} \mathrm{~F}-\mathrm{FDG}$ PET in patients with diffuse large B-cell lymphoma: SUV-based assessment at 4 cycles of chemotherapy. J Nucl Med. 2009;50:527-533.

19. Cottereau AS, Hapdey S, Chartier L, et al. Baseline total metabolic tumor volume measured with fixed or different adaptive thresholding methods equally predicts outcome in peripheral T cell lymphoma. J Nucl Med. 2017;58:276281.

20. Cottereau AS, El-Galaly TC, Becker S, et al. Predictive value of PET response combined with baseline metabolic tumor volume in peripheral T-cell lymphoma patients. J Nucl Med. 2018;59:589-595.

21. Lasnon C, Enilorac B, Popotte H, Aide N. Impact of the EARL harmonization program on automatic delineation of metabolic active tumour volumes (MATVs). EJNMMI Res. 2017;7:30 